\title{
Special educational issue on 'Basics and application of biophysical techniques in photosynthesis and related processes'
}

\author{
Johannes Messinger $\cdot$ A. Alia $\cdot$ Govindjee
}

Published online: 29 July 2009

(C) Springer Science+Business Media B.V. 2009

Due to global warming and the limited resources of (fossil) fuels on Earth, it is highly important to gain a full understanding of all aspects of how biology utilizes solar energy. The field of photosynthesis research is very broad and comprises research at various levels-from eco-systems to isolated proteins. It begins with light capture, its conversion to chemical energy, leading to oxygen evolution and carbon fixation. During almost 100 years of photosynthesis research, scientific "tools," used in this research, have grown significantly in number and complexity.

In this very first of its kind educational special issue of Photosynthesis Research, we aim to give an overview about biophysical techniques currently employed in the field. With these biophysical methods, the structures of proteins and cofactors can be resolved, and kinetic and thermodynamic information on the processes can be obtained.

All papers, no matter how complex the technique, are written by experts in the field in a way that we hope will be understood by students in biology, chemistry, and physics. In this way, these educational reviews are an important

\section{J. Messinger}

Department of Chemistry, Umeå University, 90187 Umeå, Sweden

e-mail: johannes.messinger@chem.umu.se

\section{A. Alia}

Leiden Institute of Chemistry, Leiden University, Einsteinweg 55, 2300 RA Leiden, The Netherlands e-mail: a.alia@chem.leidenuniv.nl

\section{Govindjee $(\square)$}

Department of Plant Biology, Department of Biochemistry, Center of Biophysics and Computational Biology, University of Illinois, Urbana, IL 61801, USA

e-mail: gov@illinois.edu supplement to books in the field, which we recommend for more detailed information on the present topics [see, e.g., Biophysical Techniques in Photosynthesis, edited by J. Amesz and A.J. Hoff (1995); and Biophysical Techniques in Photosynthesis, Volume II, edited by T.J. Aartsma and J. Matysik (2008), Volumes 3 and 26, respectively, in the "Advances in Photosynthesis and Respiration" series (Series Editor: Govindjee; Springer, Dordrecht)].

The biophysical techniques described in this special issue can be broadly divided into six categories: (1) Optical methods; (2) Imaging techniques; (3) Methods for determining structures of proteins and cofactors; (4) Magnetic resonance techniques for elucidating the electronic structures of protein and cofactors; (5) Theory/modeling; (6) Methods for studying substrates, products, and (redox) properties of cofactors. We had invited 50 authorities to cover these topics, and we were extremely delighted to receive 48 papers, i.e., more than $95 \%$ acceptance! These papers, which are all Educational Reviews, are being published in two parts. Part A (this issue) covers the first category: "Optical Methods." Part B will be larger in size and will cover all other categories.

Optical methods allow studying of the earliest processes of photosynthesis that occur from femtoseconds $\left(10^{-15} \mathrm{~s}\right)$ to several seconds, and even those leading to the steady-state conditions: light absorption, excitation energy transfer, primary photochemistry, regulation, and organization of the pigment-protein complexes. Light emission measurements (Fluorescence, Delayed fluorescence, and Thermoluminescence) have contributed a great deal to our understanding of the kinetics and the thermodynamics of the photosynthetic systems. Eberhard Schlodder begins this section with an Introduction to (most of) the Optical Methods used. Rudi Berera, Rienk van Grondelle, and John T.M. Kennis discuss the Ultrafast Transient Spectroscopy. 
Masayaki Komura and Shigeru Itoh present their review on Fluorescence Measurements by a Streak Camera. This is followed by a discussion of Linear and Circular Dichroism in Photosynthesis Research by Győző Garab and Herbert van Amerongen, of Resonance Raman spectroscopy by Bruno Robert, and of Infra Red (IR)/Fourier transform infra red (FTIR) spectroscopy by Catherine Berthomieu and Rainer Hienerwadel. The results of Single Molecule Spectroscopy are shown by an example of low temperature measurement on a pigment-protein complex of a purple bacterium by Silke Oellerich and Jürgen Köhler. Ulai Noomnarm and Robert M. Clegg discuss the Fundamentals and Interpretations of Fluorescence Lifetimes. Thermoluminescence (light emission monitored when we heat, in darkness, illuminated and cooled samples) has two reviews. Thermoluminescence: Experimental is covered by JeanMarc Ducruet and Imre Vass, and Thermoluminescence: Theory is covered by Fabrice Rappaport and Jérôme Lavergne. Delayed Fluorescence is presented by Vasilij Goltsev, Ivelina Zaharieva, Petko Chernev, and Reto J. Strasser. Photon Echo Studies of Photosynthetic Light Harvesting is reviewed by Elizabeth L. Read, Hohjai Lee, and Graham Fleming. And, finally Robin Purchase and Sylvia Volker present, for us, the method of Spectral Hole Burning.

Imaging methods are becoming increasingly important in the area of photosynthesis. In the imaging section, we present educational reviews on light microscopy, electron microscopy, scanning probe microscopy, and magnetic resonance imaging (MRI). The papers in this section succinctly cover basic concept of the technique and highlight applications to research in photosynthesis; they also include recent results. Egbert J. Boekema starts this section with an Introduction to Imaging Methods in Photosynthesis. Richard Cisek, Leigh T. Spencer, Donatas Zigmantas, George S. Espie, and Virginijus Barzda highlight the use of Optical Microscopy in Photosynthesis and discuss the applications of linear and non-linear optical microscopy to visualize structural dynamics inside a living cell. Three reviews cover fluorescence imaging techniques. The first review by Yi-Chun Chen and Robert M. Clegg discusses the Fluorescence Lifetime-resolved Imaging and its benefits in visualizing lifetimes of excited states. The second review is by Zdeněk Petrášek, Hann-Jörg Eckert and Klaus Kemnitz and gives a short account of Wide Field Fluorescence Lifetime Imaging Microscopy (FLIM) based on Time- and Space-Correlated Single Photon Counting (TSCSPC) to image the excited state kinetics of fluorescence molecules; this paper discusses its application in visualizing fluorescence dynamics of photosynthetic systems in cyanobacterial cells. Imaging of Fluorescence Emission from Plant Tissues is presented by Zuzana Benediktyová and Ladislav Nedbal. Exploring
Photosynthesis by Electron Tomography is reviewed by Martin F. Hohmann-Marriott and Robert W. Robertson; it summarizes its application to resolve ultrastructures of photosynthetic organisms within a few nanometers. Single Particle Electron Microscopy is presented by Egbert J. Boekema, Mihaela Folea, and Roman Kouřil. Simon Scheuring and James N. Stugis provide rationale for imaging, at high resolution, of a native photosynthetic membrane by Atomic Force Microscopy (AFM) to study supramolecular assembly of the photosynthetic complexes; Scheuring and Stugis show that AFM bridges the resolution gap between atomic structures and cellular ultrastructures. $M R I$ is a non-destructive and non-invasive technique that can be used to study the dynamics of plant water relations and water transport. Henk van As, Tom Scheenen, and Frank J. Vergeldt provide an account of MRI techniques that can be used to study plant performance in relation to its photosynthetic activity.

Structural methods can be divided into two: (1) for determining geometric structures and (2) for revealing electronic structures. For understanding how electrons are transferred within an electron transfer chain, or how chemical bonds, which are made up by electrons, are split and rearranged, information on both geometric and electronic structures are equally important for understanding the underlying design principles of unique photosynthetic catalysts. Mei Li and Wen-rui Chang, as well as James P. Allen, Chenda Seng, and Chadwick Larson describe, in two separate contributions, the basics of Protein Crystallography and X-ray Diffraction. Depending on the resolution, this approach can give very detailed information on the geometric structure of the proteins, their cofactors, and sometimes of bound substrates or products; "snapshots" are taken on deep frozen crystalline samples and provide the structural basis for understanding how proteins function. Junko Yano and Vittal Yachandra describe how X-ray Spectroscopy can be employed to obtain high-resolution data of metal-metal and metal-ligand distances in active sites of proteins without the need for crystallization of the protein. This technique and the related $X$-ray Fluorescence method described by Uwe Bergmann and Pieter Glatzel provide important information on the electronic structures of (metal) cofactors. While these X-ray spectroscopy experiments are currently mostly performed with samples frozen in different intermediate states of the catalytic cycle, kinetic X-ray spectroscopy experiments at room temperature can also be performed; these experiments have started to give important information on dynamic changes at (metal) cofactor sites. Solution structures and protein dynamics can be studied by $X$-ray Scattering (reviewed by David M. Tiede, Kristy L. Mardis, and Xiaobing Zuo) and Neutron Scattering (reviewed by Jörg Pieper and Gernot Renger). These techniques promise to give us important 
insights into how motions help to tune the energetics of biological reactions. Carsten Krebs and J. Martin Bollinger explain in their review how the combination of Rapid Freeze-Quenching and Mössbauer Spectroscopy is able to reveal structural and electronic changes occurring at iron sites during biochemical reactions.

Magnetic Resonance methods are the driving force to access photosynthesis at the molecular level. Martina Huber starts with an Introduction to Magnetic Resonance Methods in Photosynthesis. Anton Savitsky and Klaus Möbius discuss how High field EPR and its offshoots ESE (Electron Spin Echo), ENDOR (Electron-Nuclear Double Resonance), ESEEM (Electron Spin Echo Envelope Modulation), and PELDOR (Pulsed Electron Electron Double Resonance), in conjunction with site-specific isotope or spin labeling and with the support of modern quantumchemical computation methods, are capable of providing new insights into the photosynthetic transfer processes. Art Van der Est describes the application of Transient EPR to probe the geometry, electronic structure and kinetics of electron transfer in reaction centers (RCs). Gerd Kothe and Marion C. Thurnauer demonstrate What you get out of High-time Resolution EPR. They describe the quantum oscillation phenomenon observed at short delay times, after optical excitation, from the spin-correlated radical pair in photosynthetic RCs. A basic introduction to Pulsed EPR Spectroscopy is written by Maurice van Gastel. The basics as well as the recent progress on site-directed Spin Labeling $E P R$ are described by Johann P. Klare and Heinz-Jürgen Steinhoff. The application of ENDOR spectroscopy for the investigation of photosynthetic systems is reviewed by Leonid Kulik and Wolfgang Lubitz. They provide selected examples of the application of the ENDOR technique for studying stable and transient paramagnetic species, including cofactor radical ions, radical pairs, triplet states, and the oxygen-evolving complex in plant Photosystem II. Optically Detected Magnetic Resonance (ODMR) is a double resonance technique which combines optical measurements (fluorescence, phosphorescence, and absorption) with electron spin resonance spectroscopy. The basic principles of ODMR technique and some examples of application in photosynthesis are discussed by Donatella Carbonera. In the last two decades, Magic Angle Spinning (MAS) NMR has created its own niche in studies involving photosynthetic membrane protein complexes, owing to its ability to provide structural and functional information at atomic resolution. A. Alia, Swapna Ganapathy, and Huub J.M. de Groot describe the basic concept and the application of MAS NMR technique to provide us an insight into the structure and function of the Light harvesting complexes. A novel application of MAS NMR in photosynthesis research was recognized when Photo-Chemically Induced Dynamic Nuclear Polarization (Photo-CIDNP) signals were observed in bacterial RCs. We consider it remarkable that one can obtain strong NMR signals directly from the active site in all natural photosynthetic RCs even without any kind of isotopic enrichment. This effect has been revolutionizing our understanding of the electronic structure of photosynthetic RCs. Jörg Matysik, Anna Diller, Esha Roy, and A. Alia discuss the Solid-State Photo-CIDNP Effect and show that this effect has potentials which may allow for guiding artificial photosynthesis research.

Over the last several years, Theory and Modeling have gained tremendously in their capacity to provide understanding of the phenomena being investigated, and consequently in their application and impact on our field of research. Today, these theoretical tools are essential for the full interpretation of spectroscopic results, for deriving reaction mechanisms and for calculating structures and spectroscopic signatures of reaction intermediates. Our special issue contains an Overview about these methods by Francesco Buda. Then the Density Functional Theory (DFT) approach is explained by Maylis Orio, Dimitrios A. Panatazis, and Frank Neese and an introduction into the Quantum Mechanical/Molecular Mechanical (QM/MM) approach is given by Eduardo Sproviero, Michael B. Newcomer, José A. Gascón, Enrique R. Batista, and Victor S. Batista. We conclude this section with a paper by Thomas Renger on Energy Transfer Theory, which allows understanding of how antenna systems transfer absorbed solar energy to the RCs, where it is used for charge separation.

Our special issue (Part A and Part B) on Basics and Applications of Biophysical Techniques in Photosynthesis concludes with a set of papers describing Other Techniques that do not directly fall into one of the above categories, but are important for the biophysical characterization of natural and artificial photosynthesis. Gernot Renger and Bertram Hanssum summarize and explain methods for measuring Oxygen Evolution. Thermodynamic parameters of this reaction - such as enthalpy changes and apparent volume changes - can be derived by Photothermal Beam Deflection (see review by André Krauss, Roland Krivanek, Holgar Dau, and Michael Haumann, in Part B of this special issue). Katrin Beckmann, Johannes Messinger, Murray Badger, Thomas J. Wydrzynski, and Warwick Hillier describe how Membrane Inlet Mass Spectrometry can be employed for analyzing substrate-water binding in Photosystem II, characterizing carbonic anhydrase activity of photosynthetic samples and for measuring oxygen and hydrogen production of biological and artificial catalysts. Exciting ways toward Biological Hydrogen Production are outlined by Anja C. Hemschemeier, Anastasios Melis, and Thomas Happe, and finally Fraser A. Armstrong explains how Protein Film 
Electrochemistry can be utilized to characterize the reactivity of hydrogenases.

\section{Concluding comment}

The organization of this special issue on "Biophysical Techniques in Photosynthesis: Basics and Applications" began with the idea of making a special effort to further the cause of Education at a time when the Global Crisis of Energy is facing the present and future generation at an alarming rate, but our Science of Photosynthesis provides us with much hope and practical alternate solutions. We sincerely hope that this special issue of Photosynthesis Research, in two Parts (A and B), will inspire many young students to join this fascinating and rapidly developing field of research that is basic in its approach and yet offers great potential for applying the gained knowledge for the renewable production of "solar" fuels in artificial devices or in genetically modified organisms. We end this Guest Editorial with informal portraits of ourselves so that we will be recognized by others when we are at Conferences we may attend.

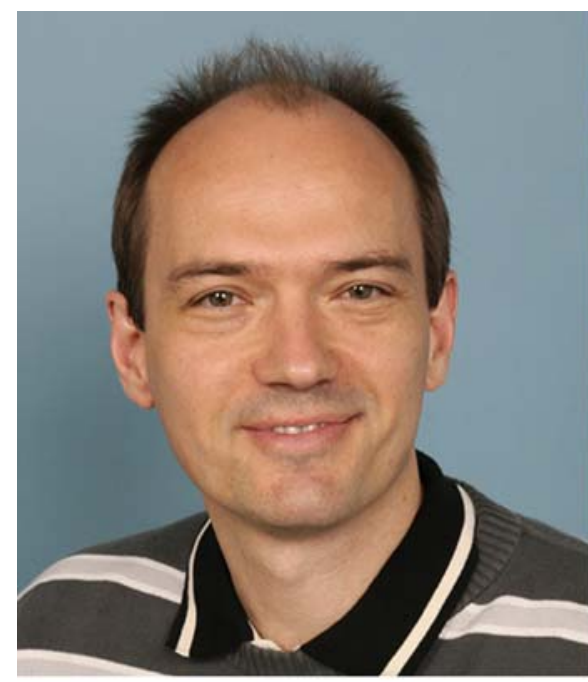

Johannes Messinger

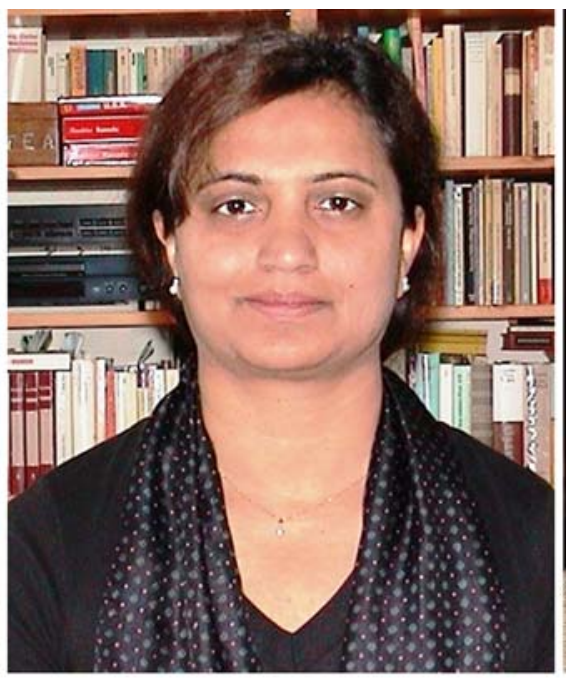

A. Alia

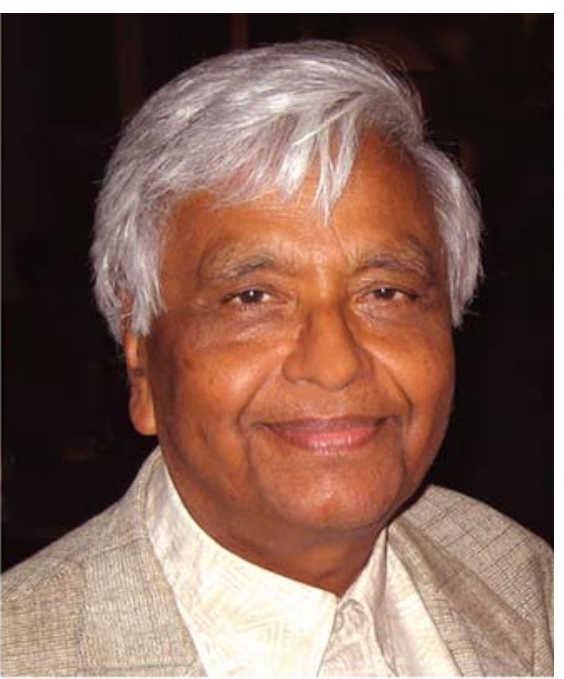

Govindjee
Acknowledgments During our editing process, each of us remembered our mentors as well as those who were, or are, associated with us, some directly related to the topic of this special issue and some not. Johannes Messinger thanks Gernot Renger, Tom Wydrzynski, Mike C. W. Evans, Jonathan H. A. Nugent, Vittal K. Yachandra, Kenneth Sauer, and Melvin P. Klein for teaching him various biophysical techniques and for being excellent mentors. Alia thanks Hans van Gorkom, Prasanna Mohanty, and Jörg Matysik for constant support and inspiration. Govindjee has a long list: he thanks his mentors Robert Emerson and Eugene Rabinowitch, and his retired, but still very active, former doctoral students George Papageorgiou, Alan J. Stemler, and Prasanna Mohanty; he has already recognized his former student Thomas J. Wydrzynski in an earlier issue of "Photosynthesis Research" (98: 13-31, 2008). In addition, Govindjee cherishes his past associations with Bessel Kok, C. Stacy French, Gregorio Weber, Herbert Gutowsky, Louis N. M. Duysens, and Don C. DeVault. All three of us are thankful to all the anonymous and notso-anonymous reviewers, David Knaff, Editor-in-Chief of Photosynthesis Research, and the following at Springer, Dordrecht (in alphabetical order): Meertinus Faber, Jacco Flipsen, Noeline Gibson, and Ellen Klink, for their excellent cooperation with us. Last but not the least, we thank the excellent Springer Corrections Team (Scientific Publishing Services (Private) Ltd (India) during the typesetting process. 